\title{
Observer Based Output Feedback Tracking Control of Robot Manipulators
}

\author{
Erkan Zergeroglu and Enver Tatlicioglu
}

\begin{abstract}
In this paper, we developed a new observer based output feedback (OFB) tracking controller for rigid-link robot manipulators. Specifically, a model independent variable structure like observer structure in conjuction with the use of desired system dynamics in the controller design have been utilized to remove the link velocity dependency of the controller and the asymptotic stability of the observer-controller couple is then guaranteed via Lyapunov based arguments. Simulation results are included to demonstrate the observer/controller performance.
\end{abstract}

\section{INTRODUCTION}

Nearly all commercially available robot manipulators do not have link velocity sensors and the ones that have velocity sensors the sensor outputs are, most of time, contaminated with noise. Therefore the output feedback tracking control of robot manipulators, where only link position information is available, have received considerable interest in robotics literature over the past years. The existing solutions to the forementioned problem can be categorized as observer based [1], [2] and filtered based [3], [4], [5], [6], [7] methods. In observer based methods either a model based [2], [8] or a model free observer is used to estimate the velocity signal, where in filtered based approaches surrogate filters are used to overcome the need of velocity measurements.

In this paper we present a new model free observer based output feedback controller, some of the past research that applied a similar approach are as follows: In [9] a variable structure output feedback controller was designed to compensate for the lack of link velocity measurement. Similarly in [10] a discontinuous controller with a high gain observer was proposed for the stabilization of a class of nonlinear systems. Recently a Luenberger like observer with an extra switching term was proposed in [11] for the output feedback control of robot manipulators.

In this paper, inspired by the observer structure given in [17], we propose a new model free observer in conjunction with a desired robot model based controller formulation for the output feedback tracking control of robot manipulators. The observer/controller structure proposed achieves semi-global asymptotic tracking despite the lack of velocity measurements. Though in its current form the proposed methodology require the exact knowledge of the system parameters, with considerably small effort adaptive and repetitive learning versions of the same observer/controller

E. Zergeroglu is with the Department of Computer Engineering, Gebze Institute of Technology, 41400, Gebze, Kocaeli, Turkey.

ezerger@bilmuh.gyte.edu.tr

E. Tatlicioglu is with the Department of Electrical \& Electronics Engineering, Izmir Institute of Technology, Urla, Izmir, 35430 Turkey.

envertatliciogludiyte.edu.tr structure can be designed to compensate for the parametric uncertainty of the robot dynamics.

The paper is organized as follows. In Section II the dynamical model of the robot manipulator with its properties used in the analysis and design of the proposed observercontroller couple are presented while Section III contains the error system development and problem formulation. In Section IV the design and stability analysis of the controller and observer are proposed. In Section V, we demonstrate the effectiveness of the proposed method through simulation results obtained from a two link, direct drive planar robot manipulator. Finally, concluding remarks are presented in Section VI.

\section{Robot Model}

The mathematical model for an $n$ DOF, revolute joint, direct drive robot manipulator is assumed to have the following form [13]

$$
M(q) \ddot{q}+V_{m}(q, \dot{q}) \dot{q}+G(q)+F_{d} \dot{q}=\tau
$$

where $q(t), \dot{q}(t), \ddot{q}(t) \in \mathbb{R}^{n}$ denote the link position, velocity, and acceleration, respectively, $M(q) \in \mathbb{R}^{n \times n}$ represents the positive-definite, symmetric inertia matrix, $V_{m}(q, \dot{q}) \in \mathbb{R}^{n \times n}$ represents the centripetal-Coriolis matrix, $G(q) \in \mathbb{R}^{n}$ is the gravitational vector, $F_{d} \in \mathbb{R}^{n \times n}$ denotes the constant, diagonal, positive-definite viscous friction matrix, and $\tau(t) \in \mathbb{R}^{n}$ represents the torque input control vector. In the subsequent development, we will assume that the left-hand side of (1) is first-order differentiable.

The dynamic model given by (1) exhibits the following properties that will be utilized in the subsequent control development and the associated stability analysis.

Property 1: The inertia matrix can be bounded from above and below by the following inequalities [13]

$$
m_{1} I_{n} \leq M(q) \leq m_{2} I_{n}
$$

where $m_{1}$ and $m_{2}$ are positive constants, and $I_{n}$ is the standard $n \times n$ identity matrix. Likewise, the inverse of the inertia matrix can be bounded as follows [13]

$$
\frac{1}{m_{2}} I_{n} \leq M^{-1}(q) \leq \frac{1}{m_{1}} I_{n}
$$

Property 2: The inertia and the centripetal-Coriolis matrices satisfy the following relationship [14]

$$
\xi^{T}\left(\frac{1}{2} \dot{M}(q)-V_{m}(q, \dot{q})\right) \xi=0 \quad \forall \xi \in \mathbb{R}^{n}
$$

where $\dot{M}(q)$ represents the time derivative of the inertia matrix. 
Property 3: The centripetal-Coriolis matrix satisfies the following relationship [8]

$$
V_{m}(q, \nu) \xi=V_{m}(q, \xi) \nu \quad \forall \xi, \nu \in \mathbb{R}^{n} .
$$

Property 4: The norm of the centripetal-Coriolis and friction matrices can be upper bounded as follows [13]

$$
\left\|V_{m}(q, \xi)\right\| \leq \zeta_{c 1}\|\xi\|, \quad\left\|F_{d}\right\| \leq \zeta_{f} \quad \forall \xi \in \mathbb{R}^{n} .
$$

where $\zeta_{c 1}$ and $\zeta_{f}$ are positive constants.

Property 5: The inertia, centripetal-Coriolis, and gravity terms in (1) can be upper bounded as follows [15]

$$
\begin{gathered}
\|M(\xi)-M(\nu)\|_{i \infty} \leq \zeta_{m 1}\|\xi-\nu\| \\
\left\|M^{-1}(\xi)-M^{-1}(\nu)\right\|_{i \infty} \leq \zeta_{m 2}\|\xi-\nu\| \\
\left\|V_{m}(\xi, \eta)-V_{m}(\nu, \eta)\right\|_{i \infty} \leq \zeta_{c 2}\|\eta\|\|\xi-\nu\| \\
\|G(\xi)-G(\nu)\| \leq \zeta_{g}\|\xi-\nu\|
\end{gathered}
$$

$\forall \xi, \nu, \eta \in \mathbb{R}^{n}$, where $\zeta_{m 1}, \zeta_{m 2}, \zeta_{c 2}$, and $\zeta_{g} \in \mathbb{R}$ are positive bounding constants, and $\|\cdot\|_{i \infty}$ denotes the induced infinity norm of a matrix.

The robot dynamics given in (1) can be written in terms of the desired trajectory in the following manner

$$
W_{d}=M\left(q_{d}\right) \ddot{q}_{d}+V_{m}\left(q_{d}, \dot{q}_{d}\right) \dot{q}_{d}+G\left(q_{d}\right)+F_{d} \dot{q}_{d}
$$

where $W_{d}\left(q_{d}, \dot{q}_{d}, \ddot{q}_{d}\right) \in \mathbb{R}^{n}$ is a function of the desired link position, velocity, and acceleration vectors, denoted by $q_{d}(t)$, $\dot{q}_{d}(t), \ddot{q}_{d}(t) \in \mathbb{R}^{n}$, respectively.

\section{PROBLEM Formulation}

The control objective is to design a link position tracking controller for the robot manipulator model given by (1) under the restrictive constraint that only the link position variable $q(t)$ is available for control development. We will quantify the control objective by defining the link position tracking error $e(t) \in \mathbb{R}^{n}$ as follows

$$
e \triangleq q_{d}-q
$$

where we assume that $q_{d}(t)$ and its first three time derivatives are bounded functions of time. To account for the unmeasurable link velocity constraint, we define $\dot{\hat{q}}(t) \in \mathbb{R}^{n}$ as the observed velocity signal. The corresponding velocity and position observation error signals $\dot{\tilde{q}}(t), \tilde{q}(t) \in \mathbb{R}^{n}$ are defined as

$$
\begin{aligned}
& \dot{\tilde{q}}=\dot{q}-\dot{\hat{q}} \\
& \tilde{q}=q-\hat{q}
\end{aligned}
$$

To ease the presentation of the analysis, we will use two auxiliary variables, filtered tracking error, denoted by $r(t) \in$ $\mathbb{R}^{n}$, and filtered observation error, denoted by $s(t) \in \mathbb{R}^{n}$ as

$$
r \triangleq \dot{e}+\alpha e, \text { and } s \triangleq \dot{\tilde{q}}+\alpha \tilde{q}
$$

where $\alpha \in \mathbb{R}$ is a positive control gain. It should be noted that, from (11), regulating $r(t)$ and $s(t)$ ensures the regulation of $e(t)$ and $\tilde{q}(t)$, respectively.

\section{OBSERVER-CONTROLler DESIGN}

Based on the subsequent error system development and the stability analysis we propose the following velocity observer

$$
\begin{aligned}
& \dot{\hat{q}}=p+K_{0} \tilde{q}-K_{c} e \\
& \dot{p}=K_{1} \operatorname{Sgn}(\tilde{q})+K_{2} \tilde{q}-\alpha K_{c} e
\end{aligned}
$$

where $p(t) \in \mathbb{R}^{n}$ is an auxiliary variable, $\operatorname{Sgn}(\cdot) \in \mathbb{R}^{n}$ is defined as

$$
\operatorname{Sgn}(\zeta)=\left[\begin{array}{lll}
\operatorname{sgn}\left(\zeta_{1}\right) & \operatorname{sgn}\left(\zeta_{2}\right) & \ldots \operatorname{sgn}\left(\zeta_{n}\right)
\end{array}\right]^{T} \forall \zeta \in \mathbb{R}^{n}
$$

with $\operatorname{sgn}(\cdot)$ being the standard signum function, $K_{0}, K_{c}$, $K_{1}, K_{2} \in \mathbb{R}^{n \times n}$ are diagonal, positive define gain matrices and $\alpha$ was defined in (11). It is straightforward to show that the time derivative of (12) yields

$$
\ddot{\hat{q}}=K_{1} \operatorname{Sgn}(\tilde{q})+K_{2} \tilde{q}+K_{0} \dot{\tilde{q}}-K_{c} r
$$

where the definition of $r(t)$ given in (11) has been utilized. Similarly, assuming that exact knowledge of all the system parameters are available, the control torque input signal $\tau(t)$ is designed to have the following form

$$
\tau=W_{d}+K_{p} e+K_{c} \alpha\left(q_{d}-\hat{q}\right)+K_{c}\left(\dot{q}_{d}-\dot{\hat{q}}\right)
$$

where the first term, $W_{d}(\cdot)$, defined in (8) is the desired robot dynamics, $K_{p} \in \mathbb{R}^{n \times n}$ is diagonal positive define control gain matrix and $K_{c}, \alpha$ were previously defined. Note that using the fact that

$$
q_{d}-\hat{q}=e+\tilde{q}
$$

the control torque input given in (15) can be rewritten in the following advantageous form

$$
\tau=W_{d}+K_{p} e+K_{c} r+K_{c} s .
$$

\section{A. Observer Analysis}

After utilizing (1) and (14), the velocity observation error dynamics can be obtained as

$$
\begin{aligned}
\ddot{\tilde{q}} & =\ddot{q}-\ddot{\hat{q}} \\
& =N_{0}-K_{1} \operatorname{Sgn}(\tilde{q})-K_{2} \tilde{q}-K_{0} \dot{\tilde{q}}+K_{c} r
\end{aligned}
$$

where the auxiliary term $N_{0}(t) \in \mathbb{R}^{n}$ is defined as

$$
N_{0}=M^{-1}(q)\left\{\tau-V_{m}(q, \dot{q}) \dot{q}-G(q)-F_{d} \dot{q}\right\} .
$$

After inserting (17) and (8) in (19), we can write $N_{0}(t)$ in the following form

$$
N_{0}=N_{d}+N_{b}
$$

where the auxiliary functions $N_{d}(t) \in \mathbb{R}^{n}$ and $N_{b}(t) \in \mathbb{R}^{n}$ are defined as

$$
N_{d}(t) \triangleq \ddot{q}_{d}
$$

and

$$
\begin{aligned}
N_{b}(t) \triangleq & \left(M^{-1}(q)-M^{-1}\left(q_{d}\right)\right) M\left(q_{d}\right) \ddot{q}_{d} \\
& +M^{-1}(q)\left\{V_{m}\left(q_{d}, \dot{q}_{d}\right) \dot{q}_{d}-V_{m}(q, \dot{q}) \dot{q}\right. \\
& +G\left(q_{d}\right)-G(q)+F_{d}\left(\dot{q}_{d}-\dot{q}\right) \\
& \left.+K_{p} e+K_{c}(r+s)\right\} .
\end{aligned}
$$


Remark 1: Exploiting the boundedness properties of the desired trajectory, we can show that $N_{d}(t)$ and $\dot{N}_{d}(t)$ are bounded. Furthermore, as illustrated in Appendix I, after using (5), (6), (7), and the mean value theorem [19], $N_{b}(t)$ can be upper bounded as follows

$$
\left\|N_{b}(t)\right\| \leq \rho_{o 1}\|e\|+\rho_{o 2}\|r\|+\rho_{o 3}\|r\|^{2}+\rho_{o 4}\|s\|
$$

where $\rho_{o i}, i=1, . ., 4$ are some positive known bounding functions that depend on the mechanical parameters and the desired trajectory and $\|\cdot\|$ denotes the Euclidean norm.

Taking the time derivative of (11) and inserting (18), the dynamics for the filtered observation error $s(t)$ can be obtained as follows

$$
\dot{s}=N_{d}+N_{b}-K_{1} \operatorname{Sgn}(\tilde{q})-K_{2} \tilde{q}-\left(K_{0}-\alpha\right) \dot{\tilde{q}}+K_{c} r
$$

and when the observer gains are selected to satisfy

$$
\alpha\left(K_{0}-\alpha\right)=K_{2}
$$

the expression in (24) can be rearranged to have the following form

$$
\dot{s}=N_{d}+N_{b}-K_{1} \operatorname{Sgn}(\tilde{q})-\frac{K_{2}}{\alpha} s+K_{c} r .
$$

Based on the expression in (26), we can state the following preliminary Lyapunov-like analysis for the observer design. Specifically, we define the following non negative scalar function, $V_{o}(t)$, as follows

$$
V_{0}=\frac{1}{2} s^{T} s+P_{0}
$$

where the scalar auxiliary function $P_{0}(t) \in \mathbb{R}$ is defined as

$$
P_{0}=\zeta_{0}-\int_{t_{0}}^{t} w_{0}(\sigma) d \sigma
$$

with the scalar function $w_{0}(t) \in \mathbb{R}^{n}$ and the non-negative constant $\zeta_{0} \in \mathbb{R}$ defined as

$$
\begin{gathered}
w_{0} \triangleq s^{T}\left[N_{d}-K_{1} \operatorname{Sgn}(\tilde{q})\right] \\
\zeta_{0} \triangleq \sum_{i=0}^{n} K_{1 i}\left|\tilde{q}_{i}(0)\right|-\tilde{q}^{T}(0) N_{d}(0)
\end{gathered}
$$

where the subscript $i=1,2, \ldots, n$ denotes the $\mathrm{i}^{\text {th }}$ element of the vector or diagonal matrix. Following a similar analysis ${ }^{1}$ to that of [16], [17], it can be proven that when $K_{1}$ satisfies the following sufficient condition

$$
K_{1 i}>\left\|N_{d i}(t)\right\|_{\infty}+\frac{1}{\alpha}\left\|\dot{N}_{d i}(t)\right\|_{\infty}
$$

where the subscript $i=1,2, \ldots, n$ denotes the $i^{\text {th }}$ element of the diagonal matrix and $\|\cdot\|_{\infty}$ denotes the $\mathcal{L}_{\infty}$ norm, then $P_{0}(t)$ of (28) is always non zero, that is $P_{0}(t) \geq 0$ and $V_{0}(t)$ is a positive-definite Lyapunov function with respect to $s(t)$ and $\sqrt{P_{0}(t)}$. After taking the time derivative of (27)

\footnotetext{
${ }^{1}$ Though the analysis very similar to that of the one given in the references, for the completeness of the presentation we have included it in Appendix II
}

and substituting (26), time derivative of (28) and (29), we can obtain

$$
\dot{V}_{0}=s^{T}\left[-\frac{K_{2}}{\alpha} s+K_{c} r+N_{b}\right] .
$$

The first term in the brackets of (31) will be used for both damping the unwanted effects of the term $N_{b}(t)$ in the composite stability analysis and to ensure the convergence of the observation error. The second term is designed to cancel out the interconnection term between the observer/controller subsystem. At this point, we are ready to proceed to the error system development.

\section{B. Error System Development}

To obtain the dynamics of $r(t)$, we take its time derivative and premultiply the resulting equation by $M(q)$, and after utilizing (1) and (9)), and performing some algebraic manipulation, to obtain

$$
M(q) \dot{r}=-V_{m}(q, \dot{q}) r+W_{s}-\tau
$$

where the auxiliary term $W_{s}(t) \in \mathbb{R}^{n}$ is defined as

$W_{s}=M(q)\left(\ddot{q}_{d}+\alpha \dot{e}\right)+V_{m}(q, \dot{q})\left(\dot{q}_{d}+\alpha e\right)+G(q)+F_{d} \dot{q}$.

After substituting the control law (17) into (32) we obtain the following closed-loop dynamics for $r(t)$

$$
M(q) \dot{r}=-V_{m}(q, \dot{q}) r+\chi-K_{c} r-K_{c} s-K_{p} e
$$

where the disturbance term $\chi(e, r, t) \in \mathbb{R}^{n}$ is defined as follows

$$
\chi=W_{s}-W_{d}
$$

with $W_{d}(\cdot)$ term was defined in (8).

Remark 2: As illustrated in [13], and also shown in Appendix I, we can exploit the boundedness properties of the desired trajectory and the properties of the robot dynamics in (5), (6), (7), to show that the norm of the variable $\chi(\cdot)$ defined in (35) can be upper bounded as

$$
\|\chi(\cdot)\| \leq \rho_{1}(e)\|e\|+\rho_{2}(e)\|r\|
$$

where $\rho_{1}(e) \in \mathbb{R}$ and $\rho_{2}(e) \in \mathbb{R}$ are known positive bounding functions. The above bound will be exploited to obtain the stability result presented in the next section.

\section{Stability Analysis}

The combination of error systems in (26) and (34) yields the following stability result for the observation error and the position tracking error.

Theorem 1: The velocity observer in (12) and the control law in (15) ensure that the closed-loop observer/controller is semi-globally asymptotically stable in the sense that

$$
\|e(t)\|,\|\dot{\tilde{q}}(t)\| \rightarrow 0 \text { as } t \rightarrow \infty
$$

provided that the controller and observer gains are selected to satisfy (25), (30), and the controller gain $K_{c}$ and the observer gain $K_{2}$ are designed as follows

$$
\begin{aligned}
& K_{c}=\left(1+\rho_{2}+k_{n} \rho_{1}^{2}\right) I_{n} \\
& K_{2}=\alpha\left(1+\rho_{o 4}+k_{n}\left(\rho_{o 1}^{2}+\rho_{o 2}^{2}+\rho_{o 3}^{2}\right)\right) I_{n}
\end{aligned}
$$


where $\rho_{1}(e), \rho_{2}(e)$ were defined in (36), $\rho_{o i}, i=1,2,3,4$ were defined in (23) and $k_{n}$ is a nonlinear damping gain selected to satisfy the following condition

$$
k_{n}>\left(1+\frac{\lambda_{2}}{\lambda_{1}}\|z(0)\|^{2}\right) / 2
$$

and $z(t) \in \mathbb{R}^{3 n+1}$ defined as follows

$$
z(t) \triangleq\left[\begin{array}{llll}
s^{T} & \sqrt{P_{0}} & r^{T} & e^{T}
\end{array}\right]^{T}
$$

and the positive bounding constants $\lambda_{1} \in \mathbb{R}$ and $\lambda_{2} \in \mathbb{R}$ are defined as

$$
\begin{gathered}
\lambda_{1}=\frac{1}{2} \min \left\{1, m_{1}, \lambda_{\min }\left\{K_{p}\right\}\right\} \\
\lambda_{2}=\frac{1}{2} \max \left\{1, m_{2}, \lambda_{\max }\left\{K_{p}\right\}\right\}
\end{gathered}
$$

Proof: We start our proof by introducing the following non negative function in the form

$$
V=V_{0}+\frac{1}{2} r^{T} M(q) r+\frac{1}{2} e^{T} K_{p} e .
$$

From (42) $V(t)$ can be upper and lower bounded as

$$
\lambda_{1}\|x\|^{2} \leq \lambda_{1}\|z\|^{2} \leq V \leq \lambda_{2}\|z\|^{2}
$$

where $x(t) \in \mathbb{R}^{3 n}$ is defined as

$$
x(t) \triangleq\left[\begin{array}{lll}
s^{T} & r^{T} & e^{T}
\end{array}\right]^{T},
$$

where $z(t)$ was defined in (40) and the positive constants $\lambda_{1}, \lambda_{2}$ were defined in (41). After differentiating (42) with respect to time, then substituting (31), (34), and cancelling common terms results in

$$
\dot{V} \leq s^{T}\left[-\frac{K_{2}}{\alpha} s+N_{b}\right]+r^{T}\left[\chi-K_{c} r\right]-\alpha \underline{\lambda}\|e\|^{2}
$$

where Property 2 was utilized and $\underline{\lambda} \in \mathbb{R}_{+}$denotes the minimum eigenvalue of $K_{p}$. After applying (23) and (35) to (45), we can form an upper bound on $\dot{V}(t)$ as follows

$$
\begin{aligned}
\dot{V} \leq & -\alpha \underline{\lambda}\|e\|^{2}-\|r\|^{2}-\|s\|^{2} \\
& +\left[\rho_{o 1}\|e\|\|s\|-k_{n} \rho_{o 1}^{2}\|s\|^{2}\right] \\
& +\left[\rho_{o 2}\|r\|\|s\|-k_{n} \rho_{o 2}^{2}\|s\|^{2}\right] \\
& +\left[\rho_{o 3}\|r\|^{2}\|s\|-k_{n}^{2} \rho_{o 3}^{2}\|s\|^{2}\right] \\
& +\left[\rho_{1}\|e\|\|r\|-k_{n} \rho_{1}^{2}\|r\|^{2}\right] .
\end{aligned}
$$

Completing the squares of the terms in the brackets we obtain

$$
\dot{V} \leq-\left[\alpha \underline{\lambda}-\frac{1}{2 k_{n}}\right]\|e\|^{2}-\left[1-\frac{1}{4 k_{n}}-\frac{1}{4 k_{n}}\|r\|^{2}\right]\|r\|^{2}-\|s\|^{2}
$$

which using the definition of $x(t)$ of (44) can be further upper bounded as follows

$$
\dot{V} \leq-\left[1-\frac{1}{2 k_{n}}\left(1+\|x\|^{2}\right)\right]\|x\|^{2} .
$$

The sign of the upper bound of $\dot{V}(t)$ is determined by the term in the brackets of (48). This term has to be positive to ensure the negative semi-definiteness of $\dot{V}(t)$, that is to ensure the negative semi definiteness of $\dot{V}(t)$ we must have

$$
1-\frac{1}{2 k_{n}}\left(1+\|x\|^{2}\right)>0 \text {. }
$$

From (43), a sufficient condition on (49) can be obtained as

$$
1-\frac{1}{2 k_{n}}\left(1+\frac{V(t)}{\lambda_{1}}\right)>0
$$

and hence at this point the analysis can be reformulated as

$$
\dot{V} \leq-\beta\|x\|^{2} \text { provided that } 2 k_{n}>\left(1+\frac{V(t)}{\lambda_{1}}\right)
$$

where $\beta \in \mathbb{R}$ is some positive constant $(0<\beta \leq 1)$. Due to the negative semi-defineteness of $\dot{V}(t)$, the maximum value that $V(t)$ can have is its initial value (i.e., $V(0)$ ), therefore, from (43), a more conservative condition on $k_{n}$ can be obtained to have the following form

$$
\dot{V} \leq-\beta\|x\|^{2} \text { provided that } 2 k_{n}>1+\frac{\lambda_{2}}{\lambda_{1}}\|z(0)\|^{2}
$$

that is when $k_{n}$ is selected to satisfy (39), we can ensure that $V(t)$ in (42) remains bounded therefore $z(t) \in \mathcal{L}_{\infty}$, thus $e(t), r(t), s(t), P_{0}(t) \in \mathcal{L}_{\infty}$. Following standard signal chasing arguments we can show that all signal in the closed loop system are bounded and $e(t)$ and $\dot{\tilde{q}}(t)$ are uniformly continuous (from the boundedness of their time derivatives), furthermore after integrating both sides of (51), it is easy to see that $x(t) \in \mathcal{L}_{2}$ and therefore $e(t), \tilde{q}(t)$, $\dot{\tilde{q}}(t) \in \mathcal{L}_{2}$. Finally, after utilizing a direct application of Barbalat's Lemma [12], we can obtain the result given in (37) provided that the gain condition of (39) is satisfied.

\section{Simulation Results}

The proposed, observer based OFB controller was simulated on a two-link, direct-drive, planar robot manipulator having the following dynamics [18]

$$
\begin{aligned}
& {\left[\begin{array}{cc}
p_{1}+2 p_{3} c_{2} & p_{2}+p_{3} c_{2} \\
p_{2}+p_{3} c_{2} & p_{2}
\end{array}\right]\left[\begin{array}{c}
\ddot{q}_{1} \\
\ddot{q}_{2}
\end{array}\right.} \\
& +\left[\begin{array}{cc}
-p_{3} s_{2} \dot{q}_{2} & -p_{3} s_{2}\left(\dot{q}_{1}+\dot{q}_{2}\right) \\
p_{3} s_{2} \dot{q}_{1} \\
f_{d 1} & 0 \\
0 & f_{d 2}
\end{array}\right]\left[\begin{array}{c}
0 \\
\dot{q}_{1} \\
\dot{q}_{2}
\end{array}\right]=\left[\begin{array}{c}
\tau_{1} \\
\tau_{2}
\end{array}\right]\left[\begin{array}{l}
\dot{q}_{1} \\
\dot{q}_{2}
\end{array}\right]
\end{aligned}
$$

where $p_{1}=3.473\left[\mathrm{~kg}-\mathrm{m}^{2}\right], p_{2}=0.193\left[\mathrm{~kg}-\mathrm{m}^{2}\right], p_{3}=$ $0.242\left[\mathrm{~kg}-\mathrm{m}^{2}\right], f_{d 1}=5.3[\mathrm{Nm}-\mathrm{sec}], f_{d 2}=1.1[\mathrm{Nm}-\mathrm{sec}]$, $c_{2} \triangleq \cos \left(q_{2}\right)$ and $s_{2} \triangleq \sin \left(q_{2}\right)$.

The simulations were performed using the following desired position trajectory

$$
q_{d}(t)=\left[\begin{array}{l}
57.30 \sin (t)\left(1-\exp \left(-0.3 t^{3}\right)\right) \\
45.84 \sin (t)\left(1-\exp \left(-0.3 t^{3}\right)\right)
\end{array}\right][\mathrm{deg}]
$$

where the exponential term was included to ensure that $\dot{q}_{d}(0)=\ddot{q}_{d}(0)=\dddot{q}_{d}(0)=0_{2 \times 1}$ and the observer/controller gains were selected as

$$
\begin{aligned}
& K_{o}=\operatorname{diag}\left\{\begin{array}{ll}
8 & 6
\end{array}\right\} \quad K_{1}=\operatorname{diag}\left\{\begin{array}{ll}
20 & 20
\end{array}\right\} \\
& K_{c}=\operatorname{diag}\left\{\begin{array}{ll}
0.012 & 0.08
\end{array}\right\} \quad K_{p}=\operatorname{diag}\left\{\begin{array}{ll}
60 & 32
\end{array}\right\} \\
& \alpha=1.2
\end{aligned}
$$

with the initial link positions selected as $q(0)=$ $\left[\begin{array}{ll}10 & 10\end{array}\right]^{T}$ deg. The link position tracking error is depicted in Figure 1, while the control torque is shown in Figure 2. From Figure 1, it is clear that the position tracking objective was met. 

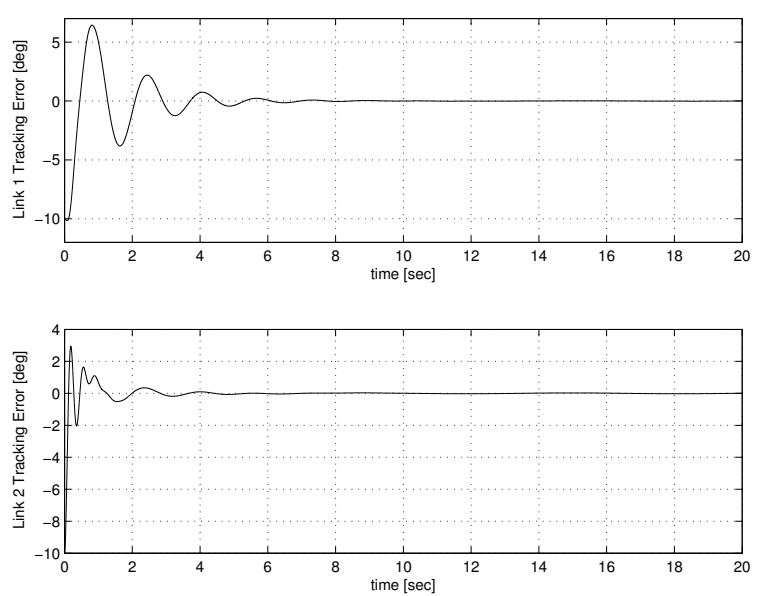

Fig. 1. Link Tracking Errors
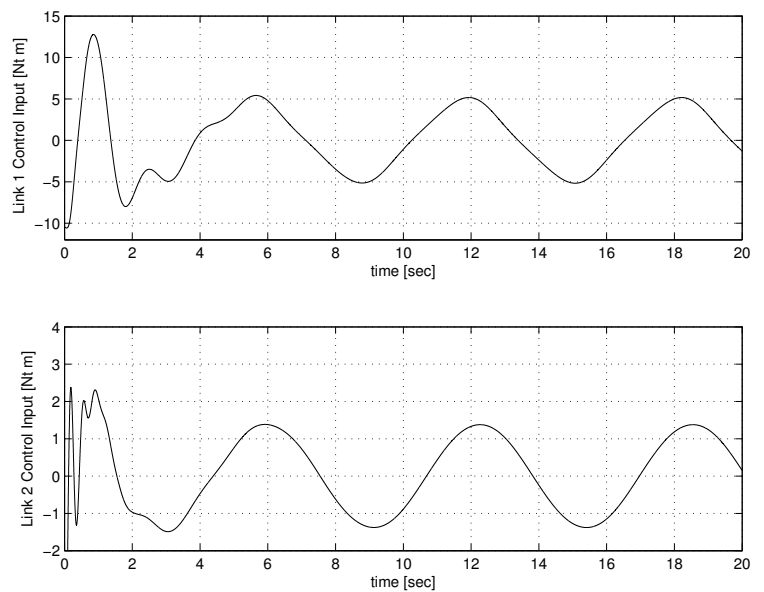

Fig. 2. Control Torque Inputs

\section{CONCLUSION}

In this paper, we have presented a new observer based output feedback tracking controller for robot manipulators. A novel observer-controller couple was introduced that ensures semi-globally asymptotic the tracking despite the lack of link velocity measurements. Simulation results are presented to illustrate the tracking performance of the observer-controller couple. Future work will focus on extending the proposed result to adaptive and learning output feedback controllers for robot manipulators.

\section{REFERENCES}

[1] S. Arimoto, V. Parra-Vega, and T. Naniwa, "A Class of Linear Velocity Observers for Nonlinear Mechanical Systems", Proc. Asian Control Conf., pp. 633-636, Tokyo, Japan, 1994.

[2] H. Berghuis and H. Nijmeijer, "A Passivity Approach to ControllerObserver Design for Robots", IEEE Tr. on Robotics and Automation, vol. 9, no. 6, pp. 740-754, 1993.

[3] T.C. Burg, D.M. Dawson, and P. Vedagarbha, "A Redesigned DCAL Controller without Velocity Measurements: Theory and Demonstration", Robotica, vol. 15, pp. 337-346, 1997.

[4] E. Zergeroglu, W.E. Dixon, D. Haste, and D.M. Dawson, "A Composite Adaptive Output Feedback Tracking Controller for Robotic Manipulators", Robotica, vol. 17, pp. 591-600, 1999.
[5] J. Yuan and Y. Stepanenko, "Robust Control of Robotic Manipulators without Velocity Measurements", Int. J. Robust and Nonlinear Control, vol. 1, pp. 203-213, 1991.

[6] F. Zhang, D.M. Dawson, M.S. de Queiroz, and W. E. Dixon, "Global Adaptive Output Feedback Control of Robot Manipulators", IEEE Tr. on Automatic Control,vol. 45, no. 6, pp. 1203-1208, 2000.

[7] E.Zergeroglu, D.M. Dawson, M.S. de Queiroz, and M. Krstic, "On Global Output Feedback Control of Robot Manipulators", IEEE International Conference on Decision and Control, pp. 5073-5078, Sydney, Australia, 2000.

[8] S. Nicosia and P. Tomei, "Robot Control by Using Only Position Measurements", IEEE Tr. on Automatic Control, vol. 35, no. 9, pp. $1058-1061,1990$

[9] C. Canudas de Wit and J. Slotine, "Sliding Observers for Robot Manipulators", Automatica, vol. 27, no. 5, pp. 859-864, 1991.

[10] S. Oh and H.K. Khalil, "Output Feedback Stabilization using Variable Structure Control”, Int. J. of Control, vol. 62, no. 4, pp.831-848, 1995.

[11] A. Abdessameud, M.F. Khelfi, "A Variable Structure Observer for the Control of Robot Manipulators", Int. J. Appl. Math. Compt. Sci., vol.16, no.2, pp. 189-196, 2006

[12] M. Krstic, I. Kanellakopoulos, and P. Kokotovic, Nonlinear and Adaptive Control Design, New York, NY, USA: John Wiley \& Sons, 1995.

[13] F.L. Lewis, C.T. Abdallah, and D.M. Dawson, Control of Robot Manipulators, New York, NY, USA: Macmillan Publishing Co., 1993.

[14] P. Tomei, "Adaptive PD Controller for Robot Manipulators", IEEE Tr. on Robotics and Automation, vol. 7, no. 4, pp. 565-570, 1991.

[15] N. Sadegh, and R. Horowitz, "Stability and Robustness Analysis of a Class of Adaptive Controllers for Robot Manipulators", Int. J. Robotics Research, vol. 9, no. 3, pp. 74-92, 1990.

[16] B. Xian, D.M. Dawson, M.S. de Queiroz, and J. Chen, "A Continuous Asymptotic Tracking Control Strategy for Uncertain Nonlinear Systems", IEEE Tr. on Automatic Control, vol. 47, no. 7, pp. 1206-1211, 2004.

[17] B. Xian, M.S. de Queiroz, D.M. Dawson and M.L. McIntyre, "Output Feedback Variable Structure-like Control of Nonlinear MEchanical Systems", Proc. of the 42nd IEEE Conference on Decision and Control, pp. 368-373, Maui, HA, USA, 2003.

[18] Direct Drive Manipulator Research and Development Package Operations Manual, Integrated Motion Inc., Berkeley, CA, USA, 1992.

[19] H. K. Khalil, Nonlinear Systems, 3rd Edition, New York, NY, USA: Prentice Hall, 2002.

\section{APPENDIX I}

\section{PROOF OF BOUNDS}

In this appendix, we illustrate how the upper bounds of $N_{b}(t)$ in (23) and $\chi(t)$ in (36) are obtained. We start with exploting the expression given in (22), which can be rewritten in the following form

$$
\begin{aligned}
N_{b}= & \left(M^{-1}(q)-M^{-1}\left(q_{d}\right)\right) M\left(q_{d}\right) \ddot{q}_{d} \\
& +M^{-1}(q)\left\{V_{m}\left(q_{d}, \dot{q}_{d}\right) \dot{q}_{d}-V_{m}\left(q, \dot{q}_{d}\right) \dot{q}_{d}\right\} \\
& +M^{-1}(q)\left\{2 V_{m}(q, \dot{e}) \dot{q}_{d}-V_{m}(q, \dot{e}) \dot{e}\right\} \\
& +M^{-1}(q)\left\{G\left(q_{d}\right)-G(q)+F_{d}\left(\dot{q}_{d}-\dot{q}\right)\right\} \\
& +M^{-1}(q)\left\{K_{p} e+K_{c} r+K_{c} s\right\}
\end{aligned}
$$

where (5) was utilized. After applying (3), (6), and (7), we can obtain an upper bound for the right-hand-side of (55) as

$$
\begin{aligned}
\left\|N_{b}(t)\right\| \leq & \frac{1}{m_{1}}\left\{\zeta_{m 1} m_{1} m_{2}\left\|\ddot{q}_{d}\right\|+\zeta_{c 2}\left\|\dot{q}_{d}\right\|\right. \\
& \left.+\lambda_{\max }\left\{K_{p}\right\}+\zeta_{g}\right\}\|e\| \\
& +\frac{1}{m_{1}}\left\{2 \zeta_{c 1}\left\|\dot{q}_{d}\right\|+\zeta_{f}+\lambda_{\max }\left\{K_{c}\right\}\right\}\|r\| \\
& +\frac{1}{m_{1}} \zeta_{c 1}\|r\|^{2}+\frac{1}{m_{1}} \lambda_{\max }\left\{K_{c}\right\}\|s\|
\end{aligned}
$$

where the fact that $\|r(t)\| \geq\|\dot{e}(t)\|$ was utilized. From the structure of (57), it is clear that the bounding functions in 
(57) are valid and $\rho_{o i}, i=1,2,3,4$ are defined as

$$
\begin{aligned}
\rho_{01} \triangleq & \zeta_{m 1} m_{2}\left\|\ddot{q}_{d}\right\|+\frac{1}{m_{1}} \zeta_{c 2}\left\|\dot{q}_{d}\right\| \\
& +\frac{1}{m_{1}} \lambda_{\max }\left\{K_{p}\right\}+\frac{1}{m_{1}} \zeta_{g}, \\
\rho_{02} \triangleq & \frac{2}{m_{1}} \zeta_{c 1}\left\|\dot{q}_{d}\right\|+\frac{1}{m_{1}} \zeta_{f}+\frac{1}{m_{1}} \lambda_{\max }\left\{K_{c}\right\} \\
\rho_{03} \triangleq & \frac{1}{m_{1}} \zeta_{c 1}, \\
\rho_{04} \triangleq & \frac{1}{m_{1}} \lambda_{\max }\left\{K_{c}\right\} .
\end{aligned}
$$

For (36), we start with the previously found bound on the same term [13] (see Chapter 6 Eq: 6.2-9) as

$$
\|\chi\| \leq \zeta_{1}\|e\|+\zeta_{2}\|e\|^{2}+\zeta_{3}\|r\|+\zeta_{4}\|r\|\|e\|
$$

where $\zeta_{i}, i=1,2,3,4$ are positive bounding constants that depend on the desired trajectory and physical parameters (i.e., link mass, link length, friction coefficients, etc.). The right-hand-side of the expression in (58) can be rewritten in the following form

$$
\|\chi\| \leq\left(\zeta_{1}+\zeta_{2}\|e\|\right)\|e\|+\left(\zeta_{3}+\zeta_{4}\|e\|\right)\|r\| .
$$

When the bounding functions $\rho_{1}(e)$ and $\rho_{2}(e)$ are selected as

$$
\begin{aligned}
& \rho_{1}(e)=\zeta_{1}+\zeta_{2}\|e\| \\
& \rho_{2}(e)=\zeta_{3}+\zeta_{4}\|e\|
\end{aligned}
$$

then the bound given in (36) is satisfied.

\section{APPENDIX II}

THE GAIN CONDITION OF $K_{1}$

In this appendix, we will illustrate how the sufficient condition of (30) is obtained. After substituting the definition of $s(t)$ in (11) into (29) and then integrating $w_{0}(t)$ in time, results in the following expression

$$
\begin{aligned}
\int_{t_{0}}^{t} w_{0}(\sigma) d \sigma= & \int_{t_{0}}^{t} \tilde{q}^{T}(\sigma) \alpha\left[N_{d}(\sigma)\right. \\
& \left.-K_{1} \operatorname{Sgn}(\tilde{q}(\sigma))\right] d \sigma \\
& +\int_{t_{0}}^{t} \frac{d \tilde{q}^{T}(\sigma)}{d \sigma} N_{d}(\sigma) d \sigma \\
& -\int_{t_{0}}^{t} \frac{d \tilde{q}^{T}(\sigma)}{d \sigma} K_{1} \operatorname{Sgn}(\tilde{q}(\sigma)) d \sigma
\end{aligned}
$$

After integrating the second integral on the right-hand side of (61) by parts, the following expression is obtained

$$
\begin{aligned}
\int_{t_{0}}^{t} w_{0}(\sigma) d \sigma= & \int_{t_{0}}^{t} \tilde{q}^{T}(\sigma) \alpha\left[N_{d}(\sigma)\right. \\
& \left.-K_{1} \operatorname{Sgn}(\tilde{q}(\sigma))\right] d \sigma+\left.\tilde{q}^{T}(\sigma) N_{d}(\sigma)\right|_{t_{0}} ^{t} \\
& -\int_{t_{0}}^{t} \tilde{q}^{T}(\sigma) \frac{d N_{d}(\sigma)}{d \sigma} d \sigma \\
& -\sum_{i=1}^{m} K_{1 i}\left|\tilde{q}_{i}(\sigma)\right|_{t_{0}}^{t} \\
= & \int_{t_{0}}^{t} \tilde{q}^{T}(\sigma) \alpha\left[N_{d}(\sigma)\right. \\
& \left.-\frac{1}{\alpha} \frac{d N_{d}(\sigma)}{d \sigma}-K_{1} \operatorname{Sgn}(\tilde{q}(\sigma))\right] d \sigma \\
& +\tilde{q}^{T}(t) N_{d}(t)-\tilde{q}^{T}\left(t_{0}\right) N_{d}\left(t_{0}\right) \\
& -\sum_{i=1}^{n} K_{1 i}\left(\left|\tilde{q}_{i}(t)\right|-\left|\tilde{q}_{i}\left(t_{0}\right)\right|\right) .
\end{aligned}
$$

The right-hand side of (62) can be upper-bounded as follows

$$
\begin{aligned}
\int_{t_{0}}^{t} w_{0}(\sigma) d \sigma \leq & \int_{t_{0}}^{t} \sum_{i=1}^{n}\left|\tilde{q}_{i}(\sigma)\right| \alpha\left[\left|N_{d i}(\sigma)\right|\right. \\
& \left.+\frac{1}{\alpha}\left|\frac{d N_{d i}(\sigma)}{d \sigma}\right|-K_{1 i}\right] d \sigma \\
& +\sum_{i=1}^{n}\left|\tilde{q}_{i}(t)\right|\left(\left|N_{d i}(t)\right|-K_{1 i}\right)+\zeta_{0} .
\end{aligned}
$$

If $K_{1}$ is chosen to satisfy (30), then it is easy to obtain the following expression from (63)

$$
\int_{t_{0}}^{t} w_{0}(\sigma) d \sigma \leq \zeta_{0}
$$

thus; from (28), it can be concluded that $P_{0}(t)$ is nonnegative. 